\title{
Power Management of Ad Hoc Routing Protocols Using Mobility Impact and Magnetic Resonance
}

\author{
Manish Bhardwaj ${ }^{*}$ Anshima Singh \\ Computer Science and Engineering Department, SRM University, Ghaziabad, India
}

Email address:

Aapkaapna13@gmail.com (M. Bhardwaj), anshima_singh@hotmail.com (A. Singh)

To cite this article:

Manish Bhardwaj, Anshima Singh. Power Management of Ad Hoc Routing Protocols Using Mobility Impact and Magnetic Resonance. Advances in Networks. Special Issue: Secure Networks and Communications. Vol. 3, No. 3-1, 2015, pp. 27-33.

doi: $10.11648 /$ j.net.s.2015030301.14

\begin{abstract}
The performance of an ad hoc routing protocol is highly dependent on the mobility model used in the ad hoc networks. Most of previous work used only Random Waypoint mobility model, which is unrealistic in many situations. The mobile nodes are often power constrained so that energy conservation is also an important issue on evaluating protocols performance. This issue can be sort out in this paper with the help of two concepts: energy conservation based on different mobility models and concept of wirelessly recharges the networks node. Most of them focused on metrics such as packet delivery ratio, delay, and route optimality. In this paper, three on demand routing protocols AODV, DSR and TORA would be evaluate at power management metrics. These protocols are simulated and compared under three different mobility models: Random Waypoint, RPGM, and Manhattan Grid and various scenarios.
\end{abstract}

Keywords: Ad Hoc Networks, Energy Conservation, And Mobility Model

\section{Introduction}

AD HOC networks are self-organizing networks composed of independent mobile nodes. There is no pre-established hierarchical infrastructure for communication between mobile nodes inside the network. All mobile nodes act as routers and route packets for each other. Providing routing mechanism in mobile ad hoc networks (MANET) has been an active research area for several years. Many routing protocols have been proposed [1]-[5] and evaluated [6] [7] over the past few years. Most of previous studies evaluate them by their performance of route selection and bandwidth usage. The metrics used are packet delivery ratio, delay, throughput, and route optimality [6] [7]. Energy conservation is also an important issue in MANET because mobile nodes are often battery powered and can not function without enough power level. As devices are being designed to be smaller (cell phones, PDAs, digital cameras), communication energy cost becomes a more significant portion of the total power consumed. In situations such as emergency rescue, military actions, and scientific field missions, energy conservation plays an even more important role which is critical to the success of the tasks performed by the network. Therefore, energy conservation should be considered carefully when designing or evaluating ad hoc routing protocols.

In reality, the performance of mobile ad hoc networks will depend on many factors such as node mobility model, traffic pattern, network topology, radio interference, obstacle positions, and so on. It is difficult to cover all these factors in simulation study of ad hoc routing protocols. In this paper we present a performance study of three ad hoc routing protocols in different mobility models, focusing on their energy conservation performance. Experiments are performed through simulations. The purpose is to identify the challenges different mobility models impose on energy conservation in ad hoc networks.

The remainder of this paper is organized as follows. Section II describes some previous work related to this topic. Section III explains the concept of wirelessly recharging of nodes. Section IV explains mobility models used in simulation in detail. Section $\mathrm{V}$ describes the simulation environment and the energy model used to calculate simulation results. Section VI presents the results and Section VII gives overall conclusions derived from the simulation results. The future research direction is described in Section VIII. 


\section{Litreture Survey}

Broch et al. [6] compared the four ad hoc routing protocols (DSR, AODV, DSDV, and TORA) in terms of packet delivery ratio, routing overhead, and path optimality. They created wireless mobile support for ns-2[13] simulation environment and implemented the four routing protocols. This work provides detailed performance analysis on ad hoc routing protocols but energy performance was not addressed and only Random Waypoint mobility model was used. Another classical performance comparison was done by Johansson et al. [7] In this work, AODV, DSDV, and DSR are compared in both random and realistic scenarios. Three carefully designed mobility scenarios are used for realistic cases: conference, event coverage, and disaster area. The performance metrics used are delay, throughput, routing overhead and average hop count. A new mobility metric are defined for characterizing node mobility by relative speeds rather than absolute speeds.

Random Waypoint is the most widely used mobility model used in evaluation of ad hoc routing protocols. It was first used by Johnson and Maltz [6] in the performance evaluation of DSR. Afterwards, it is widely used to represent mobility of nodes in mobile ad hoc networks. However, an analysis by Yoon et al. has indicated that the Random Waypoint model may produce unreliable results in simulations because the average node speed decreases over time and tend to be close to minimum speed. Besides, it is also unrealistic to model many user scenarios using Random Waypoint model.

In calculating power consumed by the network during simulation, we need an energy consumption model for the wireless network devices. Feeney has proposed a linear energy consumption model [10] for performance analysis of MANET routing protocols. Physical measuring experiments [10] [11] are done to obtain the parameters of 802.11 network interface cards. In this paper, we use the model and value of constants in to calculate the energy cost of the network.

Cano and Manzoni [12] also use ns-2 to compare the energy consumption of DSR, AODV, TORA, and DSDV. They test the routing protocols by changing different parameters of simulation environment. Although similar to this work, they used only Random Waypoint mobility model and a simpler energy consumption model.

\section{Concept of Wirelessly Power Transformation}

Household devices produce relatively small magnetic fields. For this reason, chargers hold devices at the distance necessary to induce a current, which can only happen if the coils are close together. A larger, stronger field could induce current from farther away, but the process would be extremely inefficient. Since a magnetic field spreads in all directions, making a larger one would waste a lot of energy [15]. An efficient way to transfer power between coils separated by a few meters is that we could extend the distance between the coils by adding resonance to the equation. A good way to understand resonance is to think of it in terms of sound. An object's physical structure -- like the size and shape of a trumpet -- determines the frequency at which it naturally vibrates. This is its resonant frequency [18]. It's easy to get objects to vibrate at their resonant frequency and difficult to get them to vibrate at other frequencies. This is why playing a trumpet can cause a nearby trumpet to begin to vibrate. Both trumpets have the same resonant frequency. Induction can take place little differently if the electromagnetic fields around the coils resonate at the same frequency. The theory uses a curved coil of wire as an inductor. A capacitance plate, which can hold a charge, attaches to each end of the coil. As electricity travels through this coil, the coil begins to resonate. Its resonant frequency is a product of the inductance of the coil and the capacitance of the plates [18].

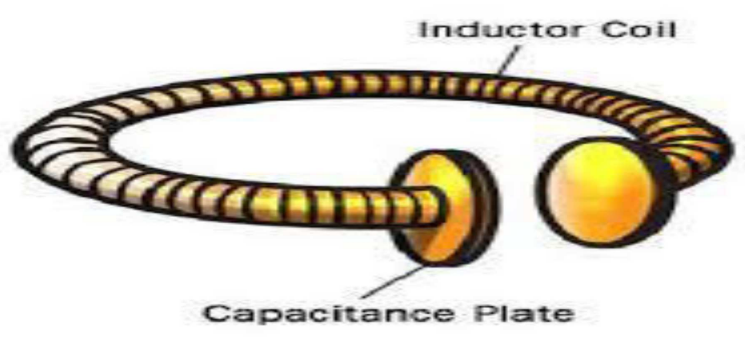

Fig. 1. Charged Coil.

Electricity, traveling along an electromagnetic wave, can tunnel from one coil to the other as long as they both have the same resonant frequency. In a short theoretical analysis they demonstrate that by sending electromagnetic waves around in a highly angular waveguide, evanescent waves are produced which carry no energy. An evanscent wave is near field standing wave exhibiting exponential decay with distance. If a proper resonant waveguide is brought near the transmitter, the evanescent waves can allow the energy to tunnel (specifically evanescent wave coupling, the electromagnetic equivalent of tunneling to the power drawing waveguide, where they can be rectified into DC power. Since the electromagnetic waves would tunnel, they would not propagate through the air to be absorbed or dissipated, and would not disrupt electronic devices. As long as both coils are out of range of one another, nothing will happen, since the fields around the coils aren't strong enough to affect much around them. Similarly, if the two coils resonate at different frequencies, nothing will happen. But if two resonating coils with the same frequency get within a few meters of each other, streams of energy move from the transmitting coil to the receiving coil. According to the theory, one coil can even send electricity to several receiving coils, as long as they all resonate at the same frequency. The researchers have named this non-radiative energy transfer since it involves stationary fields around the coils rather than fields that spread in all directions. 


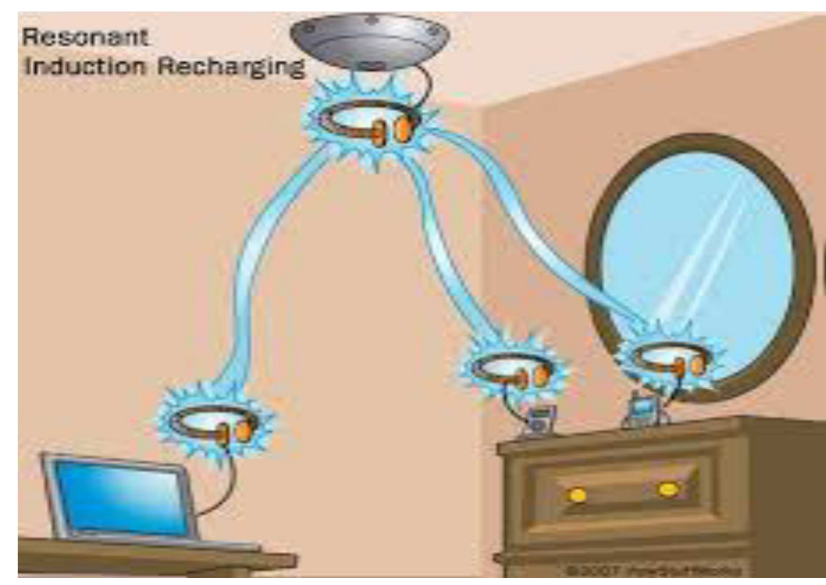

Fig. 2. Flow of charge.

According to the theory, one coil can recharge any device that is in range, as long as the coils have the same resonant frequency. "Resonant inductive coupling" has key implications in solving the two main problems associated with nonresonant inductive coupling and electromagnetic radiation, one of which is caused by the other; distance and efficiency. Electromagnetic induction works on the principle of a primary coil generating a predominantly magnetic field and a secondary coil being within that field so a current is induced within its coils. This causes the relatively short range due to the amount of power required to produce an electromagnetic field. Over greater distances the non-resonant induction method is inefficient and wastes much of the transmitted energy just to increase range. This is where the resonance comes in and helps efficiency dramatically by "tunneling" the magnetic field to a receiver coil that resonates at the same frequency. Unlike the multiple-layer secondary of a non-resonant transformer, such receiving coils are single layer solenoids with closely spaced capacitor plates on each end, which in combination allow the coil to be tuned to the transmitter frequency thereby eliminating the wide energy wasting "wave problem" and allowing the energy used to focus in on a specific frequency increasing the range.

\section{Mobility Models}

In this section, we describe the mobility models used in the simulations.

\section{A. Random Waypoint}

In Random Waypoint mobility model, parameters to be specified are pause time, minimum speed and maximum speed. Each mobile node starts from a randomly chosen position and stay for the length of pause time. When pause time expires, a destination and moving speed are randomly picked. The speed is uniformly chosen between specified maximum and minimum speed. Then the mobile node will move towards the destination with the chosen speed. Once it reaches the destination, the process of pausing, choosing destination and speed starts over again.

\section{B. Reference Point Group Mobility Model}

In RPGM model, mobile nodes are divided into groups at the beginning of the simulation. Each group has a logical center. The motion of the logical center defines the group motion. Each individual node has one reference point moving with group motion. The motion of each node is determined by two vectors: group motion vector and individual motion vector with respect to its reference point. The net motion vector of each node is the sum of the two vectors. The group motion is defined by specifying check points. Group center must follow and pass these check points. There are different ways to create various moving scenarios by changing the pattern of check points. In this work, group motion patterns are generated by Random Waypoint model. Every time the group reaches its destination, all nodes inside the group pause for a certain time and then restart the moving process. Please refer to for details about creating different group movement patterns.

\section{Manhattan Grid}

Manhattan Grid is proposed to model a city section with streets crossing each other perpendicularly. Therefore, nodes on the streets move only vertically or horizontally on the map. Each mobile node starts from a random point on certain street. The node then chooses a random destination and moves towards this destination within a predefined speed range. Upon reaching the destination, the node pauses for certain time and then repeat the process again.

\section{Simulation Environment}

The experiments performed to examine energy performance of ad hoc routing protocols on different mobility models have been done through ns-2.34 network simulator. In this section we describe the scenarios and parameters used.

A. Simulation tool and parameters

Ns-2.34 is used to compare ad hoc routing protocols over different mobility models. The underlying MAC layer protocol is defined by IEEE 802.11 standard [13]. All simulations are performed with 50 mobile nodes in a rectangular area of $690 \mathrm{~m} \times 690 \mathrm{~m}$. The length of each simulation is 500 seconds. All MAC layer operations of the wireless network interfaces are logged in trace files. Post simulation analyses are performed to each of the trace files in order to calculate the energy consumption for communication.

\section{B. Mobility \& traffic scenario generation}

BonnMotion is used to generate movement patterns for all mobility models: Manhattan Grid, RandomWaypoint and RPGM. In each model, nodes move in patterns as described in Section III accordingly.To see the speed impact on the network performance, we change the node mobility by varying the average speed in each mobility scenario. There are four speed levels: $1 \mathrm{~m} / \mathrm{s}$ (Walk), $5 \mathrm{~m} / \mathrm{s}$ (Bicycle), $10 \mathrm{~m} / \mathrm{s}$ (Motorcycle), 15m/s (Car). To provide traffic load to the ad hoc network, 10 constant bit rate (CBR) traffic streams are set up for each simulation. Each CBR traffic source sends 2 packets per second with packet size of 512 bytes. The traffic sources and destinations are chosen uniformly from all mobile nodes. It is indicated in [9] that, for RPGM, intra- 
group and inter-group traffic make significant difference in packet delivery performance. Therefore, intra-group and inter-group traffic pattern are specially generated for RPGM model to see how they affect the energy performance. In inter-group model, each pair of sender and receiver of the CBR streams are separated into different groups. In intragroup model, they are in the same group. The intra-group case of RPGM is named as "RPGM-intra" throughout this paper. Five movement cases are generated for each mobility scenario with the same parameters. All analyses are performed over the average value of the 5 cases. For the fairness of protocol comparison, each ad hoc routing protocol is run over the same set of scenarios. In total, 320 simulations are performed and analyzed.

\section{Energy consumption model}

The linear model proposed by Feeney [10] is used to calculate the energy consumption of the network. Each time a MAC layer operation takes place, certain amount of power is consumed for this operation. The energy consumption is described by the following equation:

$$
\text { Energy }=\mathrm{m} \times \text { length }+\mathrm{b}
$$

Energy: the energy cost for this operation $m$ : for incremental cost of each operation. $b$ : fixed cost of each operation length: the size of data sent/received

For example, every time the sending event occurs, we charge the battery for $b_{\text {send }}$ as a fixed cost and $m_{\text {send }}$ multiplied by the size of data sent in this event. So is the case in receiving events. For 802.11 MAC control packets (CTS/RTS/ACK), only fixed costs $b_{\text {sendct }}$, $b_{\text {recvct }}$ are charged since they all have similar size. The constants $\{\mathrm{m}, \mathrm{b}\}$ in equation (1) are obtained by physical measurements in [10] [11] for different 802.11 NICs. The values provided in [10] are used in all calculations in this paper. They are summarized in Table I.

Table 1. Constant used in simulation.

\begin{tabular}{ll}
\hline SYMBOL & VALUE \\
\hline $\mathrm{m}_{\text {send }}$ & $1.89 \mathrm{uW}-\mathrm{sec} /$ byte \\
$\mathrm{b}_{\text {send }}$ & $246 \mathrm{uW}-\mathrm{sec}$ \\
$\mathrm{m}_{\text {recv }}$ & $0.49 \mathrm{uW}-\mathrm{sec} /$ byte \\
$\mathrm{b}_{\text {recv }}$ & $56.1 \mathrm{uW}-\mathrm{sec}$ \\
$\mathrm{b}_{\text {sendetl }}$ & $120 \mathrm{uW}-\mathrm{sec}$ \\
$\mathrm{b}_{\text {recvel }}$ & 29 \\
\hline
\end{tabular}

\section{Results}

The results from the 500 simulations are presented and analyzed in this section.

From the result of all the simulations, TORA is found to be most energy consuming among the three tested protocols . Fig. 1 shows a comparison of the four protocols over Random Waypoint model. The amount of energy consumed using TORA is nearly an order of magnitude higher than the other three. The first reason is that TORA sends out too many flooding messages for route requests and updates. Every time a route condition changes, all nodes have to alter the associated height information. The second reason is that TORA updates route information very slowly. Much time is needed to flood the network with route requests and updates, so that it can not adapt fast enough to the topology changes. As a result, the network spends most of its power for the huge amount of flooding messages. Because TORA performs much worse than the other three protocols, $t$ he differences among the other three protocols become hard to tell in the graph while TORA is presented. It also makes less sense to compare protocols with an order of magnitude difference. Hence, TORA is excluded in following analyses. Fig. 2 presents the comparison of DSR, and AODV over Random Waypoint model. DSR consumes the least power at all speed levels because it requires the least amount of routing messages to adapt to the topology changes. AODV performs better at the lowest speed level because of its on-demand nature. At lower speed, the topology changes are less frequent so that AODV sends less routing messages. However, as speed goes up, more route changes are made. AODV has to generate more routing packets and consumes more power.

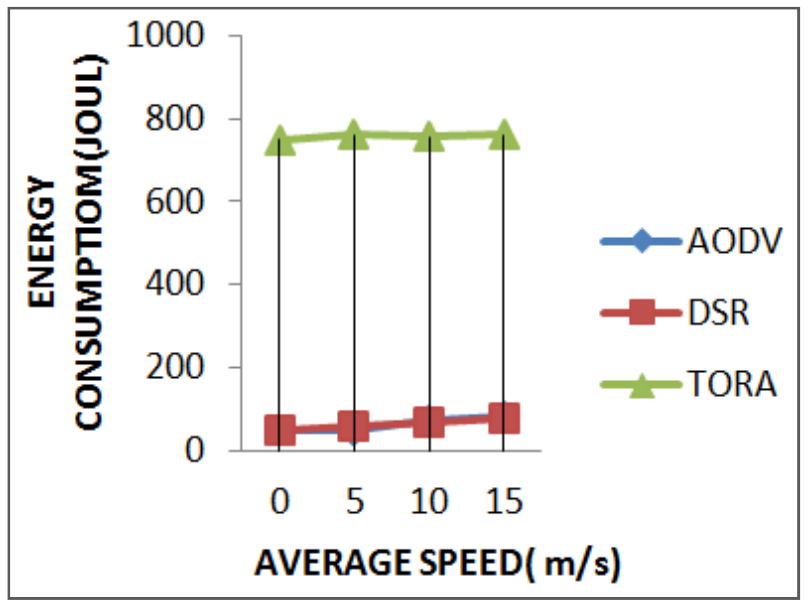

Fig. 1. Comparison of routing protocols over Random Waypoint mobility model.



Fig. 2. Comparison of routing protocols over Random Waypoint mobility model (without TORA). 
In Fig. 3, the same comparison is shown over Manhattan Grid model. In this case, DSR performs best at the lowest speed level but it becomes the worst one at the highest speed level. At higher speed, DSR and AODV perform almost equally. The energy consumption of DSR and AODV both grow with speed rapidly. This result shows that on-demand protocols are more sensitive to speed of mobile nodes than proactive protocols. The explanation is that higher speed causes more route changes which force ondemand protocols to generate routing messages more frequently. Fig. 4 shows the result over RPGM mobility model. In this case, nodes move in groups and this pattern reduces the rate of topology change. This reduction of topology change is directly reflected in the amount of energy consumed by each protocol.

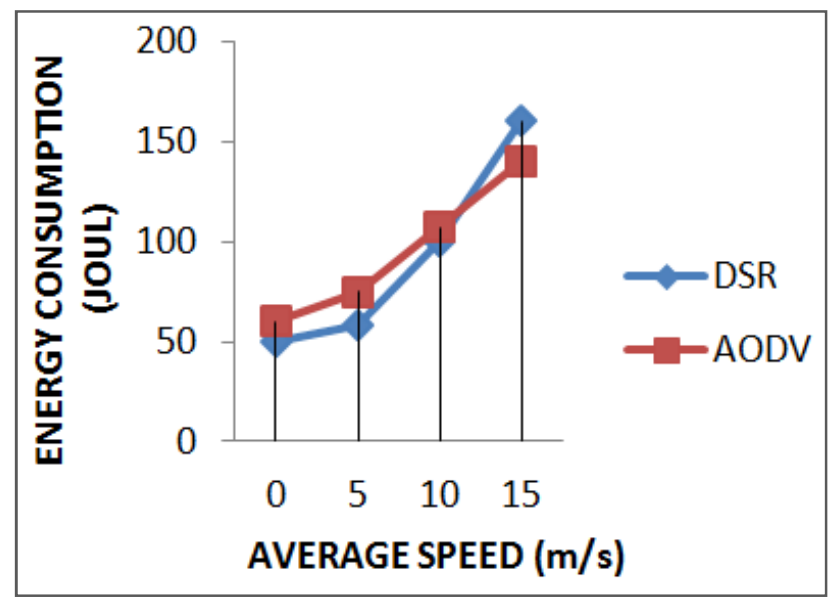

Fig. 3. Comparison of routing protocols over Manhattan Grid mobility model (without TORA).

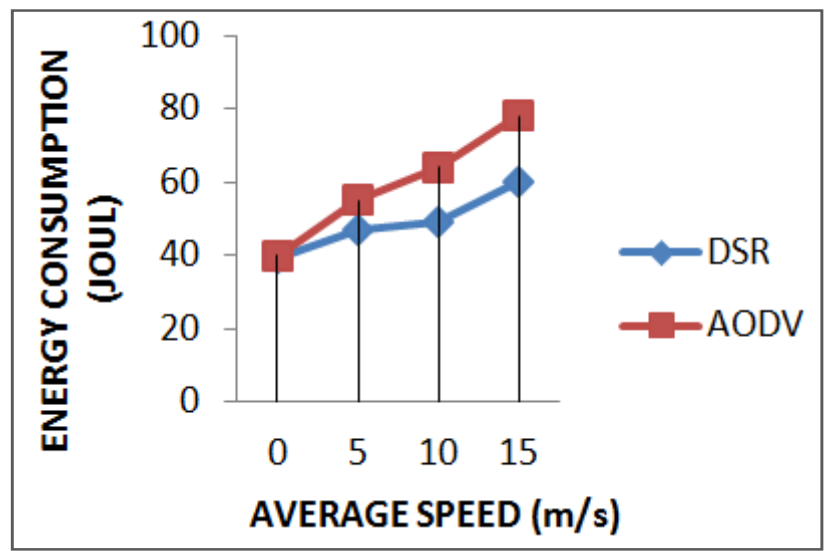

Fig. 4. Comparison of routing protocols over RPGM mobility model (without TORA).

Fig. 5 compares the performance of each protocol over RPGM model with intra-group traffic. In this ideal environment, on-demand protocols DSR and AODV perform much better than proactive protocol. Group mobility model with local traffic constitutes an extremely friendly environment for DSR and AODV.

Fig. 6 presents the comparison of DSR performance over the four mobility models. It is obviously shown that DSR is quite sensitive to node speed in Random Waypoint and Manhattan Grid model. Comparing the four mobility models, Manhattan Grid is the most challenging environment for DSR. DSR is much more sensitive to node speed in Manhattan Grid than any other model. In RPGM with intragroup traffic, DSR consumes very low power and becomes independent of node speed. It is because local traffic insulates DSR from topology changes among groups.

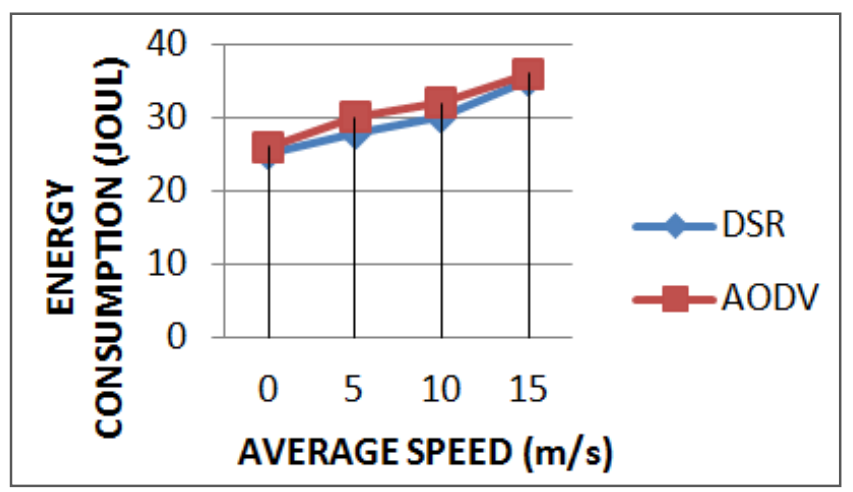

Fig. 5. Comparison of routing protocols over RPGM mobility model with intra-group traffic (without TORA).

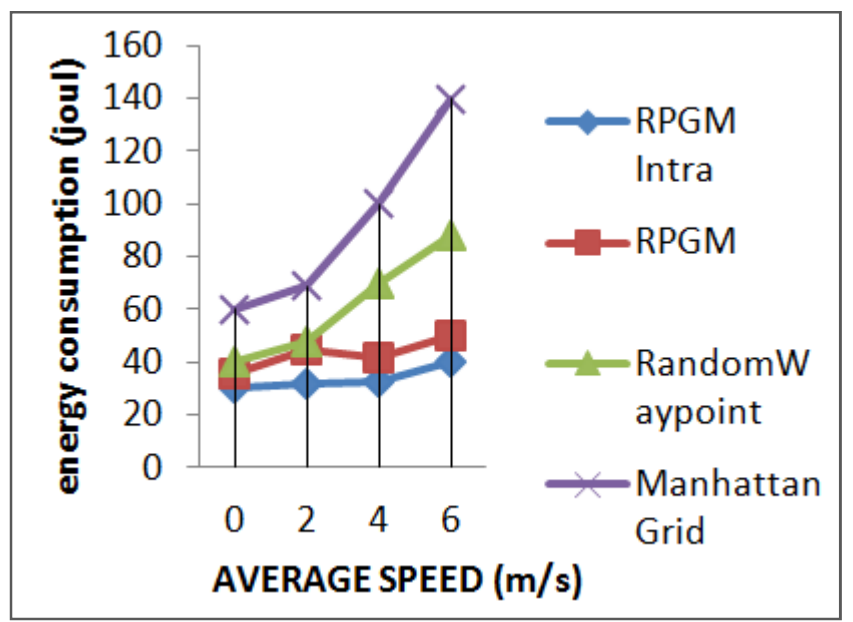

Fig. 6. Effect of mobility models on DSR.

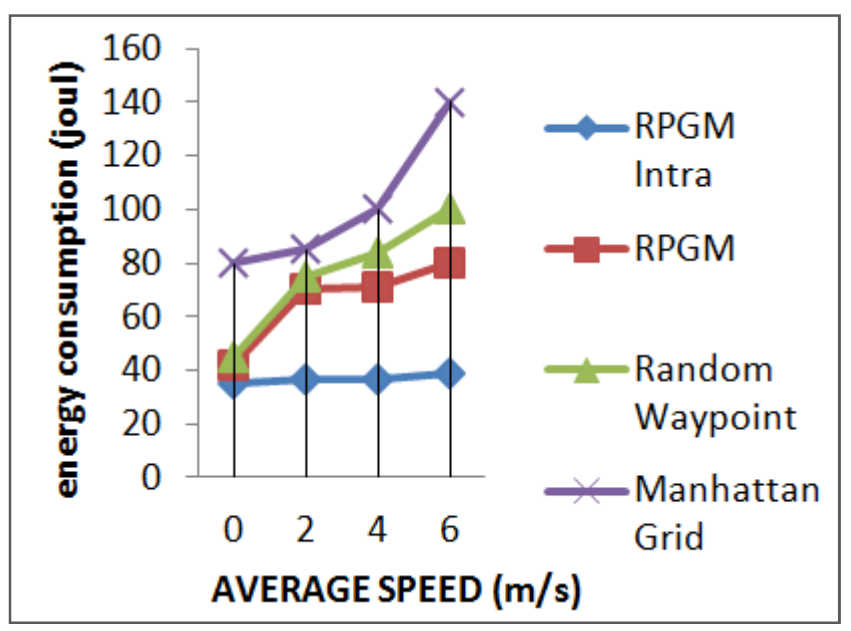

Fig. 7. Effect of mobility models on AODV. 
The performance of AODV in different mobility models is presented in Fig. 7. Similar to DSR, AODV consumes most power in Manhattan Grid. However, the sensitivity to node speed here is less than DSR. Except for the fact that the energy consumed by AODV is generally higher than DSR, the challenges imposed by mobility models to AODV are similar to those of DSR. It is reasonable because AODV and DSR are both on-demand protocols and the only major difference is that DSR uses source routing rather than distance vector in AODV.

\section{Conclusions}

The following conclusions are drawn from the results of this work.

1. The performance of ad hoc routing protocols greatly depends on the mobility model it runs over.

2. Reactive protocols are more speed-sensitive while proactive protocols are not.

3. Routing in Ad hoc networks over Manhattan Grid environments is more challenging than Random Waypoint and RPGM.

4. In situations where nodes move in groups, on-demand protocols perform better than proactive ones in terms of energy conservation.

5. DSR performs best among the evaluated protocols, except for high speed cases in Manhattan Grid model.

These facts are useful when deploying ad hoc networks with power constrained devices. When the network is used in a low speed environment, DSR is generally the best choice for energy conservation. However, in high speed cases, the movement pattern of the network should be further considered to determine whether to choose an on-demand or proactive protocol. If the network is used in a group-based moving environment, on-demand protocols are better choices In a highly dynamic environment such as Manhattan Grid, the performance of on-demand protocols decreases and proactive protocols save more power. Although not directly pointed out in the analyses, it is worth mentioning that the flooding approach used to disseminate query information is very expensive in energy cost. The network will be in the risk of consuming most of its power on flooding messages with fast topology changes and heavy packet traffic load. Thus, reducing the use of flooding approach is another important issue in designing an energy conserving protocol for ad hoc networks.

\section{Future Work}

In this work, the emphasis is on evaluating the amount of energy needed to route the same traffic using different protocols over different mobility models . However, the nodes often have finite battery power and the batteries will be drained and this event causes route breaks. This factor is not taken into account in this work. In the future, we would like to investigate the impact of the battery dying effect to the network performance. Besides, it is shown that network traffic pattern also plays an important role in energy performance when comparing the cases of RPGM and RPGM-intra. Hence, applying different traffic patterns and investigate its relation to energy performance is worthy of more exploration. Furthermore, there are other low power protocols developed to minimize the network power consumption, such as in [22]. Evaluation of these protocols is another topic for future analysis work.

\section{References}

[1] David B. Johnson, David A. Maltz, Yih-Chun Hu, "The Dynamic Source Routing Protocol for Mobile Ad Hoc Networks (DSR)," Internet Draft, IETF Mobile Ad hoc Networks (MANET) Working Group.

[2] Charles E. Perkins, Elizabeth M. Belding-Royer, Samir R. Das, "Ad hoc On-Demand Distance Vector (AODV) Routing," Internet Draft, IETF Mobile Ad hoc Networks (MANET) Working Group.

[3] C. Perkins, P. Bhagwat, "Highly Dynamic DestinationSequenced Distance-Vector Routing (DSDV) for Mobile Computers," in Proc. ACM SIGCOMM'94, London, UK, 1994.

[4] Vincent D. Park and M. Scott Corson, "A Highly Adaptive Distributed Routing Algorithm for Mobile Wireless Networks", in Proc. of IEEE INFOCOM '97, Kobe, Japan, Apr. 1997.

[5] Feeney, L.M., "A Taxonomy for Routing Protocols in Mobile Ad Hoc Networks”, in SICS Technical Report T99:07, 1999

[6] Josh Broch, David A. Maltz, David B. Johnson, Yih-Chun Hu, Jorjeta Jetcheva ,"A Performance Comparison of Multi-Hop Wireless Ad Hoc Network Routing Protocols," in Proc. MobiCom'98, Dallas, TX, Oct. 1998

[7] Per Johansson, Tony Larsson, Nicklas Hedman, Bartosz Mielczarek, Mikael Degermark, "Scenario-based performance analysis of routing protocols for mobile ad-hoc networks," in Proc. MobiCom'99, Seattle, Washington, 1999.

[8] David B. Johnson and David A. Maltz," Dynamic Source Routing in Ad Hoc Wireless Networks," in Mobile Computing, (ed. T. Imielinski and H. Korth), Kluwer Academic Publishers, 1996.

[9] S. Shah, E. Hernandez, and A. Helal, "CAD-HOC: A CAD Like Tool for Generating Mobility Benchmarks in Ad-Hoc Networks," in Proc. of the second IEEE/IPSJ Symposium on Applications and the Internet (SAINT'02), Nara, Japan, 2002.

[10] Feeney, L.M., “An energy-consumption model for performance analysis of routing protocols for mobile ad hoc networks," Mobile Networks and Applications Vol. 3, No. 6, June 2001.

[11] Feeney, L.M., and Nilsson, M., "Investigating the energy consumption of a wireless network interface in an ad hoc networking environment," in Proc. IEEE INFOCOMM, Anchorage AK, April, 2001.

[12] Juan-Carlos Cano, Pietro Manzoni, "A Performance Comparison of Energy Consumption for Mobile Ad Hoc Network Routing Protocols, " in Proc. IEEE/ACM MASCOTS 2000: Eighth International Symposium on Modeling, Analysis and Simulation of Computer and Telecommunication Systems, San Francisco, USA, August 2000. 
[13] IEEE Computer Society LAN MAN Standards Committee, "Wireless LAN Medium Access Control and Physical Layer Specificationd," in IEEE 802.11 Standard, Aug. 1999.

[14] Joshua L. Jabs, C. Hwa Chang and Robert Tingley, "Performance of a Very Low Power Wireless Protocol," in Proc. IEEE Globe Com '01, San Antonio, TX, USA, 2001.

[15] Manish Bhardwaj. Selection of Efficient Relay for EnergyEfficient Cooperative Ad Hoc Networks. American Journal of Networks and Communications. Special Issue: $\mathrm{Ad} \mathrm{Hoc}$ Networks. Vol. 4, No. 3-1, 2015, pp. 5-11. doi: 10.11648 j.ajnc.s. 2015040301.12

[16] Megha Sharma, Shivani Rohilla, Manish Bhardwaj. Efficient Routing with Reduced Routing Overhead and Retransmission of Manet. American Journal of Networks and Communications. Special Issue: Ad Hoc Networks. Vol. 4, No. 3-1, 2015, pp. 22-26. doi: 10.11648/j.ajnc. s. 2015040301.15

[17] Manish Bhardwaj, Enhance life Time of Mobile Ad hoc Network using WiTri City and Backpressure Technique, 18770509 (C) 2015 The Authors. Published by Elsevier B.V. This is an open access article under the CC BY-NC-ND license, doi: 10.1016/j.procs. 2015.07.447.

[18] A. Kurs, A. Karalis, R. Moffatt, J. D. Joannopoulos, P. Fisher, M. Soljacic. 2008. Wireless Power Transfer via Strongly Coupled Magnetic Resonances. Science. http://www.sciencemag.org/cgi/rapid.df/1143254?ijkey=94ff. Ay4jRMqU\&keytype=ref\&siteid=s ci (accessed December 5, 2011). 